\title{
Reshaping Classics With Joint Efforts Home and Abroad The Chinese-English Edition of Hawke's Version of Hongloumeng
}

\author{
Full Lin Chen ${ }^{1, a, *}$, Keqiang Liü,b
}

${ }^{1}$ School of Foreign Languages, Anhui University of Technology, Ma'anshan, Anhui, China

${ }^{2}$ Foreign Language College, Honghe University, Mengzi, Yunnan, China

amaggicl@126.com, blc1971@qq.com

Keywords: Hongloumeng, The Story of The Stone, the Chinese-English version.

\begin{abstract}
Forty years after The Story of The Stone, one of the classics of the English translation of Hongloumeng was published by the Penguin Group in the 20th century, the year of 2012 witnessed its Chinese-English version published by the Shanghai Foreign Language Education Press. With joint efforts contributed by the scholars home and abroad, the translation greeted the world with a brand new appearance. The new version has not only made a big breakthrough in terms of the original versions consulted by David Hawkes in his translating, but it has also created a new academic space for the study of the English translation of Hongloumeng. The thesis introduces it in detail from such aspects as its appearance, its contents, and its academic values.
\end{abstract}

\section{中西合力 重塑经典 一《红楼梦》霍译汉英对照本 \\ 陈琳 $1, a,{ }^{*}$ ，刘克强2,b \\ 1安徽工业大学外国语学院, 马鞍山, 安徽, 中国 \\ 2红河学院外国语学院, 云南, 蒙自, 中国 \\ amaggicl@126.com, blc1971@qq.com}

关键词：《红楼梦》; 霍译; 汉英对照

中文摘要. 继 20 世纪英国企鹅出版社发行《红楼梦》霍译本 40 年之后, 上海外语教育出版社 于2012年首度发行霍译汉英对照本。在中外学者共同努力下, 《红楼梦》英译经典以崭新面 目重新问世。对照本在霍译底本方面取得重大突破, 同时也为《红楼梦》英译研究提供新的 学术生长点。本文从外观、内容、学术价值等方面, 系统介绍霍译汉英对照本。

\section{1. 引言}

自1816年英国传教士Robert Morrison翻译《红楼梦》第三十一回片段开始， 《红楼梦》 西渐历经两个世纪, 经历片段翻译、节译、选译、转译等多种形式, 直至 20 世纪下半叶出现 英文全译本, 其中最为引人注目的当属《红楼梦》杨宪益戴乃迭译本 (后文简称杨译) 和霍 克斯闵福德译本（后文简称霍译）。但是，由于《红楼梦》原著版本众多成书过程复杂，大 师为译界提供丰富素材同时, 也铸造了一柄红楼译评的达摩克利斯之剑。换句话说, 译者参 照原著底本的神秘和缺场, 给红楼译评埋下隐患（至少在结构主义视域下如此）。无论杨译 底本 “有正本程乙本” 与 “庚辰本程甲本” 之争，还是更为扑朔迷离的霍译 “百衲本” 猜测, 《红楼梦》英译研究中达摩克利斯之剑始终高悬若隐若现。杨译本率先突破重围。作为大中 
华文库系列丛书之一, 国内先后发行《红楼梦》汉英对照两个版本, 为英译研究者提供可据 参照的蓝本。在闵福德先生和范圣宇先生几载的辛勤努力下, 上海教育出版社终于在2012年7 月1日出版发行《红楼梦》霍译汉英对照本 (以下简称霍译对照本)。本文从外观、内容、学 术价值等方面, 对霍译对照本进行详细介绍, 以飨读者。

\section{2. 霍译对照本外观与内容介绍}

与其他译者不同, 霍克思以原著故事情节为线索将《红楼梦》分为五卷。霍译五卷本的 编排是译者主体性突出表现之一，霍译对照本亦保留这一体制特色分为五卷册。

\section{1 霍译对照本外观介绍}

霍译对照本为 16 开精装本, 朱砂红套色, 装帧典雅质感温馨。每册封面右上方菱形方框 内写有书名、作者, 以及卷册编号。封面上方有大写的霍译本书名、每卷册汉英对照名, 以 及译者姓名, 其中卷册译名均统一为四字格表述, 其中第壹卷《枉入红尘》, 第式卷《海棠

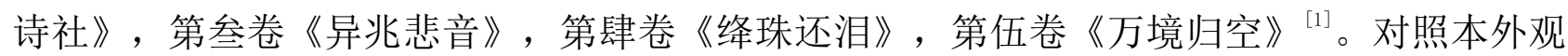
图文并茂, 封面及书内均附有著名红楼画家戴敦邦先生的精美插图。就封面图片来说, 第壹 卷《枉入红尘》图片选自第三回, 第式卷《海棠诗社》图片选自第三十七回, 第参卷《异兆 悲音》图片选自第七十五回，第肆卷《㖓珠还泪》图片选自第八十七回，第伍卷《万境归空》 图片选自第一百二十回。此外，前言、附录、回目标题下方均配有相关插画，譬如第一回回 目标题 “甄士隐梦幻识通灵 贾雨村风尘怀闺秀” 下方配图为 “贾雨村风尘怀闺秀” ，第二回 回目标题 “贾夫人仙逝扬州城 冷子兴演说荣国府” 下方配图为 “贾夫人仙逝扬州城” 等, 文 字与插图相得益彰, 共同阐释文本主题。同时, 与霍译旧版不同的是, 对照本内所有韵文文 字, 包括回目标题和诗词韵文, 均用彩色高亮标出。

\section{2 霍译对照本内容介绍}

与企我出版社发行的《红楼梦》霍译五卷本相比, 霍译对照本在所有项目上均增添对应 译文, 包括目录、序言、附录, 以及正文内容等。同时, 第壹卷新增闵福德先生特意为对照 本撰写的《序言》, 还有范圣宇先生撰写的《校勘说明》。这两段文字就对照本编写初衷和 整个过程, 以及体例编纂、操作标准等具体细节进行详介。总体来说, 霍译对照本至少在以 下三个方面可圈可点, 即尽力复原霍克思参照底本、适度修订英译文、调整修改序跋汉译文。

\subsection{1 复原底本}

霍克思先生在生前最后一段岁月里，以《红楼梦英译笔记》为重要线索，积极筹备 “复 原” 自己的底本 (被称为 “霍本” ), 闵福德和范圣宇先生也为此付出巨大努力, 耐心细致 校对每一回, 特别注意其中细节。霍译对照本中文部分出现三种符号值得关注。其一, 用尖 括号く>表示文字参校他本, 譬如第一回霍译本有Wu Yu-feng called it A Dream of Golden Days, 但在所有校对本中均无此句, 所以放入尖括号内, 即 “〈至吴玉峰题曰《红楼梦》。>”。其 二, 用方括号[]表示 “原文被有意删去, 或者是译者漏译的部分” ，譬如第二回 “宁公居长, 生了两个儿子。宁公死后......” , 霍译本只出现Ning-guo was the elder of the two. When he $\operatorname{died} \cdots$, 显然 “生了两个儿子” 属于有意删除或者漏译部分, 所以放入方括号内, 即 “宁公 居长, [生了两个儿子。] 宁公死后……”。其三, 用花括号 \{\} 表示 “霍克思综采众长, 纠正作 者的叙事破绽，或者竟是他自己的独创”，譬如第三回 $\{$ 紫鹃 $\}$ 、第九十三回 $\{$ 铁槛寺 $\}$ 和 $\{$ 水月 庵\}等。当然, 有时也会出现上述两种符号套用的情况, 譬如第一回 “<至脂砚斋 [甲戌]抄阅 再评, 仍用《石头记》。>”，表明整句话参校他本，其中 “甲戎” 被有意删除或漏译 ${ }^{[1]}$ 。 2.2.2 修订霍译 
《红楼梦》汉英对照本以上世纪旧版为蓝本，参照霍克思先生《红楼梦英译笔记》及相 关日记和书信, 对现有译文作了全面系统校订。那么, 霍译对照本与旧译相比出现了哪些变 动？我们将上述两个版本进行对比，发现其中呈现一些体例变化，至少表现在以下三点。 其一, 标点符号变动, 包括逗号和连字符等, 譬如对照本第九十七回The bed, where she sat on it, seemed to dig into her ‥, 此句两个逗号在旧版中没有出现; 再如The other womenfolk accompanied him as far as the inner gate before turning back一句中, womenfolk在旧版中带有连 字符, 书写形式为women-folk ${ }^{[1]}$ 。其二, 新增星号*。星号在霍译旧版中通常出现在空行前, 成为较大意义段落的起论标记。第九十七回Next day, Xi-feng came over after breakfast一句前, 旧版只有空行, 而对照本在空行前新增* ${ }^{[1]}$ 。其三, 霍译对照本出现脚注形式, 譬如Stone's Note to Reader分别出现在第十八、五十三、六十三回，旧版均为正文内容，但对照本只有第十八 回仍为正文文字，第五十三回和第六十三回均移入脚注 ${ }^{[1]}$ 。

除了体例有些变更外, 还出现一些词汇更改, 譬如第九十七回, 旧版为Xi-feng watched him carely, 对照本则为Xi-feng watched him carefully, 其间出现carely与carefully的差异 ${ }^{[1]}$ 。针对霍 译全文词频变化情况, 我们利用EditPlus软件统计得出数据显示, 霍译对照本和旧版相比变动 幅度不大, 旧版共852963词, 对照本共852709词, 120回总体删减 254词, 平均每章修改两个 单词 $(254 / 120=2.11)$ 。且以前 20 回为例, 章回词频变化仅为个位数的占7回, 分别是第七、 八、十、十一、十二、十五、十八回。当然, 每个章回究竟有哪些词汇发生具体变化, 还有 待进一步研究。下图提供霍译两个版本词频变化总体趋势:

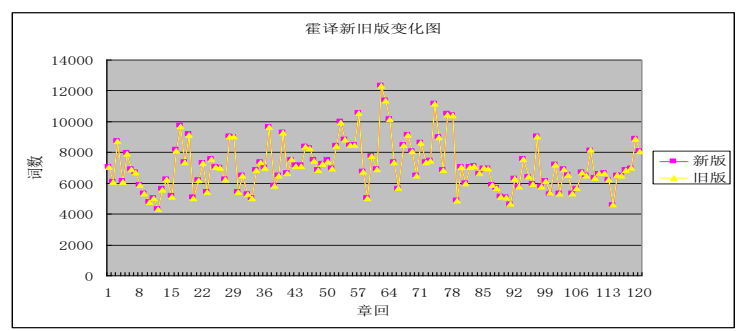

图1 霍译新旧版变化图

\subsection{3调整范译}

范圣宇先生曾将霍译五卷本序跋全部翻译完毕 ${ }^{[2]}$, 但在霍译对照本中，译文经过多处调 整和修改。首先, 词藻择选更加考究, 譬如曹雪芹曾经担任过schoolmaster, 原译文为 “校长”, 对照本改为 “学堂训导”，显然后者与当时历史文化语境更为贴合。此外，大量四字格的使 用使得译文表述在符合译入语表达习惯基础上，更显华丽练达。其次。句式编排重新调整， 有的地方改动幅度较大。限于篇幅, 我们从第壹卷 “附录” 和 “译者序” 中选取部分段落加 以说明。且以附录中对林黛玉薛宝钗合用判词的解释为例 ${ }^{[1]}$ :

[例1] The picture is a simple rebus. Two trees make up the Chinese character for 'Lin' , whilst 'jade belt' is an inversion of Dai-yu' : the 'Dai' of Dai-yu' s name really means 'eye-black' , but it sounds the same as the word for 'belt', and yu means 'jade'. The pile of snow is a rebus of Bao-chai's surname Xue, which sounds the same as the Chinese word for 'snow'...

[旧版译文] 画是很简单的画谜。两株树组成了中文里的“林” 字，而 “玉带” 是 “黛玉” 倒过来: “黛玉” 的 “黛” 指的是画眉的墨, 但读起来像是 “带”。“玉” 的意思是 “宝玉”。 那堆雪隐藏着宝钗的姓 “薛” ……

[对照本译文] 那幅画是一个很简单的画谜。两棵树组成了中文里的“林” 字，而“玉带” 则是把 “黛玉”的读音倒过来：“黛玉” 的 “黛” 指的是 “画眉之墨”，但它与 “带” 同音， 而 “玉” 的意思则是 “玉石”。那堆雪是宝钗的姓 “薛” 的画谜, “薛”与中文的 “雪” 发 音相同。 
上述两段译文对比来看，对照本译文比旧版表述更加确切。譬如 “那幅画”比“画”指 代明确, “倒过来” 前增添 “读音” 两字, 表述不再笼统, 而 “宝玉”一词因与原著男主人 公同名, 改为 “玉石” 可以避免一些不必要的误解。相比之下, 最后一句改动幅度最大, 对 照本译文不仅比旧版表述更加具体，而且贴近原文。此外，小处修缮譬如 “画眉的墨” 四字 在对照本中添加引号, 显示译文修改细致入微。

\section{3. 霍译对照本学术价值}

国内《红楼梦》译评，以1929年吴宓先生发表于《大公报》文学副刊上的《王际真英译 节本〈红楼梦>述评》拉开序幕, 自上世纪80年代红楼译评逐渐升温并引发热潮。新世纪以来 文章数量猛增, 论著频频面世, 探究范围逐步扩大, 成果卓著蔚为大观。与此同时, 有学者 认为红楼英译研究出现 “瓶颈” , 陷入 “两大困境” : 其一, “如何实现对比研究的可比性”, 即杨译本, 尤其是霍译本的研究, 如何处理其底本为 “百衲本” 的问题。显然, 《红楼梦》 英译研究达摩克利斯之剑被再度高举。其二, “如何发现新的研究点” ${ }^{[3]}$ 。红楼译评重复选 题、重复研究现象较为严重, 如何突破现有研究并发现新的生长点, 是目前面临的紧迫任务 ${ }^{[4]}$ 。霍译对照本的问世, 不仅在其前始终莫衷一是的霍译底本方面取得重大突破, 同时也为

《红楼梦》英译研究提供新的学术生长点。依笔者浅见, 霍译对照本至少在如下三个方面为 红楼译评开辟新的探索空间。其一, 底本深入挖掘。霍译对照本对可能参照的原著底本进行 初步校对梳理, 那些 “不详细注出采自何种本子” 的不下 348 处。因此, 余下工作以及由此引 发的翻译探讨有待学者进一步挖掘 ${ }^{[3]}$ 。其二, 霍译新旧版本对比研究。本文利用相关软件, 仅就霍译新旧版本词频做初步考察, 但究竟增删了哪些? 为什么要增删? 效果如何? 诸如此 类问题, 还有待进一步研究探讨。其三, 译者主体性深度探寻。对于红楼译评者来说, 对照 本中标注的译者有意删除漏译, 以及独创部分非常具有研究价值。据我们统计, 译者有意删 除或漏译不下93处, 译者独创部分不下 117 处。那么, 这些有意删除漏译和独创部分与译者遵 循的伦理道德具有怎样联系? 这些不旁为有红楼英译探究中颇有价值的话题。

\section{4. 结束语}

自英国企鹅出版社首次发行《红楼梦》霍译本之后, 上海外语教育出版社在新世纪发行 霍译对照本, 两位译者一一霍克思和闵福德先生对原有译文字㽎句酌, 精益求精, 而校勘者 一范圣宇先生不仅仔细修缮汉译, 更在底本探寻中付出巨大努力, 中西合力重塑经典。虽 然那柄达摩克利斯之剑并未完全落地, 霍译底本的神秘莫测甚至连霍克思本人也难辨真伪, 但被誉为 “绝代双璧” 的霍译对照本无疑为 《红楼梦》英译研究揭开新的一页, 在底本鉴别 方面取得重大突破同时, 也为红楼译评提供新的学术生长点。我们期待着在翻译理论蓬勃发 展, 翻译越来越呈现跨学科和界面性的今天, 红楼译坛群芳吐芯, 不断开拓崭新学术空间。 揭秘与探究不仅是《红楼梦》原著, 也是《红楼梦》译本, 更是红楼译评的永恒魅力所在。

\section{References}

[1] Xueqin Cao. The Story of The Stone. Trans., David Hawkes \& John Minford. Shanghai foreign Language Education Press (2012).

[2] Shengyu Fan. Limited Outlook of Hongloumeng. Chinese Social \& Scientific Publishing House (2004).

[3] Shiyang Ran \& Yang Ping. Bottleneck and Breakthrough in the Chinese-English contrastive study of Hongloumeng. China Publishing Journal. vol.12, pp. 61-63, 2013.

[4] Shicong Liu. Translation and Evaluation of Hongloumeng. Nankai University Press (2004). 